\title{
GAMMA-RAY BURSTS AS THE DEATH THROES OF MASSIVE BINARY STARS
}

\author{
Ramesh Narayan ${ }^{1}$, Bohdan Paczyński ${ }^{2}$, and Tsvi Piran ${ }^{1,3}$
}

\begin{abstract}
It is proposed that gamma-ray bursts are created in the mergers of double neutron star binaries and black hole neutron star binaries at cosmological distances. Bursts with complex profiles and relatively long durations are the result of magnetic flares generated by the Parker instability in a post-merger differentially-rotating disk. Some bursts may also be produced through neutrino-antineutrino annihilation into electrons and positrons. In both cases, an optically thick fireball of size $\lesssim 100 \mathrm{~km}$ is initially created, which expands ultrarelativistically to large radii before radiating. Several previous objections to the cosmological merger model are eliminated. It is predicted that $\gamma$-ray bursts will be accompanied by a burst of gravitational radiation from the spiraling-in binary which could be detected by LIGO.
\end{abstract}

Subject Headings: Accretion — Black Holes — Gamma Rays: Bursts — Gravitation — Magnetic Fields - Neutrinos — Pulsars — Stars: Binaries — Stars: Neutron — Stars: Supernovae - X-rays: Binaries

\section{Submitted to Ap J (Lett.), March 24, 1992}

${ }^{1}$ Harvard-Smithsonian Center for Astrophysics, Cambridge, MA 02138

${ }^{2}$ Princeton University Observatory, Princeton, NJ 08544

${ }^{3}$ Racah Institute for Physics, The Hebrew University, Jerusalem, Israel 


\section{INTRODUCTION}

Recent results obtained with the Burst and Transient Source Experiment (BATSE) on the Compton Gamma-Ray Observatory (Meegan et al. 1992) suggest strongly that gamma-ray bursts (see Higdon \& Lingenfelter 1990 for a review) originate at cosmological distances. The 153 bursts reported so far appear to be isotropic in the sky and to have a distribution of $V / V_{\max }$ that is consistent with a cosmological population extending to redshifts $z \sim 1$ (Mao \& Paczyński 1992, Piran 1992). The required event rate is $\sim 10^{-6} \mathrm{yr}^{-1}$ per $L^{*}$ galaxy, and the typical energy released in a burst is $\sim 10^{51}$ erg. The short rise times observed imply a source size $\sim 100 \mathrm{~km}$.

Based on these requirements, many authors (Paczyński 1986, Goodman 1986, Eichler et al. 1989, Piran 1990, Narayan, Piran \& Shemi 1991, Paczyński 1991, Piran, Narayan \& Shemi 1992) have suggested that $\gamma$-ray bursts arise in the merger of binaries consisting of either two neutron stars (NS-NS) or a black hole and a neutron star (BHNS). This suggestion is attractive for a number of reasons. (1) The model employs a known source population; four NS-NS radio pulars have been discovered in the Galaxy, viz. PSR 1534+12, PSR 1913+16, PSR 2127+11C, and PSR 2303+46. (2) The scenario invokes orbital decay through the emission of gravitational radiation, for which we have direct observational evidence in the case of PSR1913+16 (Taylor \& Weisberg 1989); moreover, three of the above four pulsars have merger times shorter than the Hubble time. (3) In a merger, an energy $>10^{53}$ erg will be released in a time $\sim 1 \mathrm{~ms}$ and within a radius $<100 \mathrm{~km}$, satisfying the observational constraints. (4) NS-NS and BH-NS mergers are estimated to occur at the rate of $\sim 10^{-6}-10^{-5} \mathrm{yr}^{-1}$ per galaxy (Narayan, Piran \& Shemi 1991, Phinney 1991), in good agreement with the observed burst rate. 
Many arguments have been made against cosmological scenarios in general and the merger model in particular. Among these, one objection appears at first sight to be quite serious. If $10^{51} \mathrm{erg}$ of $\gamma$-rays are created in a volume of size $100 \mathrm{~km}$, the optical depth due to $\gamma+\gamma \rightarrow e^{+}+e^{-}$will be extremely large and the photons will apparently be trapped (Schmidt 1978). This objection was refuted by Paczyński (1986) and Goodman (1986) who showed that an optically thick ball of energy, a "fireball," will expand relativistically and thereby radiate most of its energy when it becomes optically thin. Because of relativistic beaming, a distant observer receives a burst of radiation whose temperature and duration will be similar to the initial temperature and initial light-crossing time of the fireball. Relativistic beaming also circumvents the so-called "Ruderman limit" (Ruderman 1975), which sets an upper limit to the distance of a source for a given source temperature, flux, and variability timescale. (Beaming solves a related problem in the case of extragalactic radio jets).

Although the problems associated with the large optical depth and the Ruderman limit have been solved, several other objections remain. (1) How is the energy converted into $\gamma$-rays? (2) How can one avoid baryon contamination which will significantly modify the evolution of the relativistic fireball? (3) How can one obtain bursts with a median duration $\sim 10 \mathrm{~s}$ when the dynamical timescale is $\lesssim 1 \mathrm{~ms} ?$ (4) Why are burst profiles so complex and individually unique? (5) Why do some bursts have a precursor several seconds before the primary burst (Murakami et al. 1991)? (6) What produces the power-law $\gamma$-ray spectrum, which extends well beyond the pair creation limit of $511 \mathrm{keV}$ and sometimes even up to a few hundred MeV? (7) How can one explain the cyclotron absorption features seen in a few bursts? (8) What is the explanation for the redshifted electron-positron annihilation lines claimed in some bursts? (The 
evidence for these lines is not very strong, cf. Messina \& Share 1992.) (9) Why have no galaxies been found in the vicinities of bright $\gamma$-ray bursts with well-determined positions (Schaefer 1990)?

The aim of this paper is to describe a qualitative scenario for the production of $\gamma$-ray bursts in mergers of NS-NS and BH-NS binaries, and to demonstrate that there are plausible solutions to most of these objections.

\section{MERGER SCENARIO}

The progenitors of close NS-NS and BH-NS binaries must be massive X-ray binaries consisting of an $\mathrm{O}$ or $\mathrm{B}$ main sequence star and a neutron star or a black hole (e.g. Vela X-1, Cyg X-1, cf. Trimble 1991 for a review). When the main sequence star evolves, the binary very likely undergoes a common envelope phase, after which one has a tight binary consisting of the helium core of the OB star and its compact companion. Cyg X-3 $\left(P_{\mathrm{b}}=4.79 \mathrm{hr}\right)$ appears to be an excellent example of this stage of evolution (van Kerkwijk et al. 1992).

We are interested in Cyg X-3 like binaries with separations $a_{\mathrm{o}}$ ranging from $\sim$ few $\times 10^{10} \mathrm{~cm}$, the radius of the helium core (assuming that it behaves like a helium main sequence star), to $\sim$ few $\times 10^{11} \mathrm{~cm}$, the limit beyond which gravitational radiation losses in the double degenerate binary phase are too slow to cause a merger within the age of the universe. To make a NS-NS binary, the helium core needs to have a mass $M_{\mathrm{He}}$ between $\sim 2.5 M_{\odot}$ (in order to have a supernova explosion) and $4.2 M_{\odot}$ (in order to leave behind a bound NS-NS binary, assuming a symmetric supernova explosion and a neutron star mass of $\left.1.4 M_{\odot}\right)$. After the explosion, we are left behind with a NS-NS binary with an orbital eccentricity $e=\left(M_{\mathrm{He}} / 2.8\right)-0.5$, periastron 
separation $a_{\mathrm{p}}$ equal to the pre-explosion separation $a_{\mathrm{o}}$, and a recoil velocity $v_{\text {recoil }}=$ $180\left(M_{\mathrm{He}}-2.8\right)\left(M_{\mathrm{He}}+1.4\right)^{-1 / 2}\left(a_{\mathrm{p}} / 10^{11} \mathrm{~cm}\right)^{-1 / 2} \mathrm{~km} \mathrm{~s}^{-1}$. Similar estimates for a BH-NS binary give somewhat lower orbital eccentricities and space velocities.

For typical numbers, the recoil velocity of the binary is large enough for the system to escape from a small galaxy (though probably not from an $L^{*}$ galaxy). There is growing evidence recently that the blue extragalactic light is dominated by faint dwarf galaxies between $22^{m}-24^{m}$ (Cowie 1991, and references therein). These galaxies are at modest redshifts $(z \sim 0.2-0.4)$ and are very numerous $(1$ and 6 per square arcmin at $22^{m}$ and $24^{m}$ ). If supernovae are strongly correlated with the blue light, then the majority of compact binaries are probably born in low-mass galaxies, escape from their hosts, and move distances of up to $1 \mathrm{Mpc}$ in a few $\times 10^{9}$ yr before they merge. The lack of obvious host galaxies associated with $\gamma$-ray bursts is then not surprising. The burst positions are likely to be offset from that of their parent galaxies by up to an arc minute, and at this separation the association will be confused by several other faint galaxies in the field.

The typical duration of a $\gamma$-ray burst is $\sim 10 \mathrm{~s}$, and the time delay between the precursor and main pulse is also $\sim 10 \mathrm{~s}$ (Murakami et al. 1991). For a NS-NS binary in a near-circular orbit, the time to merge is given by $t_{\text {merge }}=38\left(a / 10^{7.5} \mathrm{~cm}\right)^{4} \mathrm{~s}=$ $160\left(P_{\mathrm{b}} / 0.1 \mathrm{~s}\right)^{8 / 3} \mathrm{~s}$. Although the efficiency of tidal interactions and spin-up of the two stars as they spiral towards each other is uncertain (Haensel, Paczyński \& Amsterdamski 1991, Kochanek 1992), it is likely that there will be sufficient heating produced by tidal dissipation to lead to a super-Eddington luminosity. This may produce some of the precursors. The luminosity may also drive a wind from the surfaces of the two stars, creating slow-moving baryonic material which may block $\gamma$-ray emission from 
the compact binary. On the other hand, a later-produced relativistically expanding burst may be able to shock on the slow wind to produce non-thermal radiation. Yet another possibility is that the precursors may in fact be due to the early phases of the relativistic burst material plowing through the pre-burst wind.

Once the two stars merge, there will be $\sim$ few $\times 10^{53}$ erg of energy divided moreor-less equally into three forms: (1) thermal energy, (2) ordered rotational energy, and (3) "non-uniform" kinetic energy (either chaotic motions or differential rotation). The third form of energy may be quite significant, and may be located quite far from the center, if there is strong splashback during the merger as may happen if the rotations of the two stars are not synchronized with the orbit. Each of the three energy forms will be released through a different channel.

The thermal energy is radiated mostly as neutrinos and antineutrinos. Goodman et al. (1987) showed that in Type II supernovae the finite cross-section associated with the reaction, $\nu+\bar{\nu} \rightarrow e^{+}+e^{-}$, results in $\sim 10^{51}$ erg being converted into electromagnetic energy. Eichler et al. (1989) proposed that this mechanism may produce $\gamma$-ray bursts in binary mergers. The timescale of the process is the neutrino cooling time, $\sim$ few seconds.

Although modest variability may be expected in neutrino emission because of the chaotic (possibly convective) flows in the post-merger fluid, the complicated and highly variable time structure observed in some bursts may be too extreme to be explained by the neutrino process alone. We suggest that the neutrino mechanism produces the subclass of bursts with relatively smooth profiles and time durations $\sim$ few seconds (e.g. GB830801b, Fig. 2 in Higdon \& Lingenfelter 1990), and possibly also some precursors.

The rapid rotation of the post-merger object will almost certainly cause the fluid to be dynamically unstable, leading to rapid loss of energy and angular momentum 
on a timescale of a few msec. This instability dies down when the ratio of kinetic to gravitational energy falls below $\sim 0.27$. At this stage the rotating system will collapse directly to a black hole if the total mass is greater than the maximum mass of a neutron star (note that some stiff equations of state permit neutron stars more massive than $2.8 \odot)$, and if the angular momentum $J$ is smaller than $G M^{2} / c$. If $J c / G M^{2}>1$, collapse is temporarily prevented, but the system will continue to exhibit a secular instability through which it will lose angular momentum on a timescale of a few seconds (Friedman 1983).

Most of the energy released during the first dynamical stage will be in the form of gravitational radiation, which could be of importance to future gravitational radiation detectors, but we do not expect any direct electromagnetic signal. During the second secular phase there may be coherent emission of gravitational radiation that could be detected by LIGO, but other processes like neutrino cooling and build up of the magnetic field will also take place.

A fraction of the original mass will not participate directly in the central instability and collapse, but will form a surrounding disk/torus which may itself undergo dynamical instabilities (e.g. Narayan \& Goodman 1989). We consider it likely that after this phase a significant amount of residual kinetic energy, $\lesssim 10^{52} \mathrm{erg}$, will survive in a relatively long-lived differentially-rotating disk, and we believe that this energy can be tapped almost entirely through electromagnetic processes.

In the merger of a BH-NS binary, the neutron star will be tidally disrupted, with some of the matter being swallowed by the black hole, and the remainder forming a torus, generating heat and neutrinos in the process. Subsequent evolution may proceed in a similar fashion in both the BH-NS and NS-NS scenarios, and it is not clear at this time which of the two is more efficient as a $\gamma$-ray burster. 
The mechanism we propose to produce $\gamma$-ray bursts similar to GB820331 and GB841215 (Fig. 2 in Higdon \& Lingfelter 1990), which have complex time profiles, is based on magnetic fields. Immediately after the merger the field strength is probably $\sim 10^{12} \mathrm{G}$, but instabilities such as the Balbus \& Hawley (1991) mode will cause the field to build up quickly to something like an equipartition value $\sim 10^{16}-10^{17} \mathrm{G}$. Even if there is no instability, the shearing action of the differential rotation will lead to field build-up on a slower timescale $\sim 10^{4}$ rotations. (Note that, while electric fields are limited to a maximum strength of $\sim 10^{13} \mathrm{~V} \mathrm{~cm}^{-1}$ by the pair creation threshold, magnetic fields can build up to much higher strengths.) Once the field achieves equipartition, it will tend to exhibit the Parker (1966) instability in which the field will float up and break out of the disk (relativistically in our case) on a dynamical time, leaving the matter behind. Particularly near the top of the magnetic loop, the baryonic contamination may be quite low, making the conditions favorable for a gamma-ray burst. The burst itself will probably be created as a series of explosive reconnection events in the rising magnetic field, just as in solar flares. For the assumed field strength, the temperature of the flare will be $\sim$ few $\times 10^{11} \mathrm{~K}$, the photons will have characteristic energies $\sim 10 \mathrm{MeV}$, and the total energy in a single burst will be $\sim 10^{50}$ erg for linear dimensions $\sim 1-10 \mathrm{~km}$. The resulting optically thick fireball will expand relativistically as described by Paczyński (1986), Goodman (1986), Shemi \& Piran (1990), and Paczyński (1990).

A very important feature of the model is that the rate of energy release is not limited by the Eddington luminosity, but by the efficiency with which the Parker instability can separate magnetic fields from matter. The energy dissipation occurs above the disk, and, because of the relativistic outward motion of the flare, very little 
energy returns to the disk. If this aspect of the scenario works efficiently (which needs to be demonstrated), then we have a viable solution to the problem of how to separate the photons from the baryons (how to "separate light from the darkness").

The flare activity will make the burst profile quite complex, with many subbursts. Also, the total duration over which the successive sub-bursts occur will be quite variable from one object to another, depending on the details of the post-merger fluid configuration. These features eliminate some of the strongest objections to the merger scenario. The important point is that the model has two distinctly different timescales: firstly, a dynamical time, $\sim 10^{-3} \mathrm{~s}$, which may be related to the rise times of individual flares (because the Parker instability, once it gets going, operates essentially on a dynamical time), and, secondly, an accretion (or magnetic "viscosity") time, $\sim 1-100 \mathrm{~s}$, related to the duration of the whole $\gamma$-ray burst. We cannot calculate the second timescale from fundamental theory, but we note that in other objects with disks, such as cataclysmic variables and X-ray binaries, the accretion time is typically many orders of magnitude longer than the dynamical time.

A generic problem with any cosmological model is that the huge initial optical depth leads to themalization of the fireball (Paczyński 1990) and a black body spectrum. This is in clear conflict with the observed spectra of $\gamma$-ray bursts, which have a significant excess of X-rays and very hard $\gamma$-rays compared to any Planck spectrum. There are at least two possible solutions to this problem, both related to the flarelike energy release and rapid variability. First, while the instanteneous spectrum of a local flare may be Planckian, the superposition of many Planck curves with a wide range of temperatures and intensities may appear as a very broad broken power law. Second, it is likely that the flares will eject some mass due to imperfect separation of 
energy and matter. These relativistic ejecta may collide with each other, as well as with the pre-merger wind, at a fairly large radius and at low optical depths, giving a non-Planckian spectrum through various non-thermal processes. For instance, given the strong magnetic fields, synchrotron processes might naturally produce the observed power-law spectrum if enough relativistic particles are present.

An important point to note is that the observed photon energy $h \nu_{\text {obs }}$ is related to the emitted energy in the frame of the radiating fireball $h \nu_{\mathrm{em}}$ by the large relativistic $\gamma \sim 10^{2}-10^{3}$ of the expansion (Paczyński 1986, Goodman 1986). Therefore, there is no difficulty in producing very hard spectra. In particular, note that a cut-off at 511 $\mathrm{keV}$ in the emitter frame translates to a cut-off at $\gamma \times 511 \mathrm{keV}$ for the observer, which can correspond to several hundred $\mathrm{MeV}$.

The fact that the energy release in the model is predominantly through a magnetic process may solve another potential problem, the cyclotron absorption lines that have been claimed in some bursts. In very rough terms, an initial field of $10^{16} \mathrm{G}$ at the point of initiation of the burst will be diluted to a field strength $\sim 10^{10} \mathrm{G}$ in the frame of the emitting fluid if the expansion factor is $\sim 10^{3}$. However, because of relativistic beaming, the observed cyclotron line will appear as if it is emitted in a field $\sim \gamma \times 10^{10}$ $\mathrm{G} \sim 10^{13} \mathrm{G}$, which is roughly consistent with the claimed field strengths (Murakami et al. 1988, Fenimore et al. 1988). The narrowness of the observed lines requires a stable value of the Lorentz $\gamma$ for the duration of the line. This will be difficult to maintain for any length of time in our highly erratic burster, in qualitative accordance with the "now you see them, now you don't" nature of the reported lines.

Another interesting possibility should be finally mentioned. As the disk cools through neutrino emission, it might at some point make a transition to a superconducting superfluid state. Plasma processes in a degenerate superconducting superfluid 
have been hardly studied at all. It is conceivable that the separation of the field and the baryons may be particularly efficient in a magnetic superfluid. This problem merits further investigation.

\section{CONCLUSIONS}

We propose a scenario for gamma-ray bursts involving merging NS-NS and BH-NS binaries at cosmological distances. In our picture, complex main bursts with highly variable time profiles are produced through many successive magnetic flares and reconnection events tapping the free energy in differential rotation in a post-merger disk. Less variable bursts may be produced by neutrinos converting a fraction of their energy into electromagnetic radiation. The precursors of the bursts may be produced by the neutrino mechanism, or by the initial phases of the post-merger ultra-relativistic burst ejecta plowing through the slow wind created in the pre-merger tidal heating phase, or even possibly by the pre-merger heating itself. This scenario is capable of explaining the qualitative features of most of the observations, and circumvents many of the arguments against the cosmological merger model of gamma-ray bursts.

The model employs two kinds of objects, NS-NS and BH-NS binaries, and two distinct mechanisms, magnetic flares and neutrino interactions. Different combinations of these may have distinct observational signatures which could be potentially identified in the large database of burst profiles being acquired by BATSE.

Apart from $\gamma$-rays, it is likely that the bursts in our scenario will also eject $\sim$ $10^{51}$ erg of energy in ultra-relativistic ejecta, i.e. cosmic rays, with little or no nonrelativistic matter. These ejecta should create a supernova-like remnant far from the parent galaxy, where the density of diffuse matter may be very low. 
The scenario makes a definite prediction. If and when LIGO is commissioned (Vogt 1992), strong $\gamma$-ray bursts should be accompanied by gravity wave detections (though the reverse need not necessarily be true, particularly if the $\gamma$-rays are beamed). Since the strength of the gravitational radiation signal from a pre-merger binary can be calculated accurately (Lincoln \& Will 1990) except for the unknown inclination, LIGO should provide good distance estimates to individual bursts and also pinpoint the exact time of the merger. This information will permit more detailed interpretation of the $\gamma$-ray burst observations.

The scenario we have presented in this paper is at this point no more than a qualitative sketch of some likely possibilities. The purpose here is merely to show that none of the many arguments against the merger model are necessarily fatal. More detailed analyses of the individual ideas are needed now.

One other point worth emphasizing is that the scenario is in a real sense the most conservative one possible at the present time. Based purely on the observations, a cosmological location for $\gamma$-ray bursts is the most reasonable hypothesis; all other proposals need coincidences at some level in the properties of the associated source population. Within the cosmological framework, our scenario employs the most obvious source population that we can think of, double compact binaries. This population is definitely known to exist, we know its members will merge, and we can be certain that huge quantities of energy will be released. However, even if this particular model turns out to be incorrect, the cosmological hypothesis itself will survive, given the observed distribution of burst positions and intensities.

We thank Roger Blandford and John Friedman for discussions and comments. This work was supported by NASA grants NAG 5-1901 and NAG 5-1904. 


\section{REFERENCES}

Balbus, S. A. \& Hawley, J. F. 1991, ApJ, 376, 214

Cowie, L. L. 1991, in Primordial Nucleosynthesis and Evolution of Early Universe, Astrophysics \& Science Library, Vol. 169, p. 425 (Eds.: K. Sato \& J. Audouze, Kluwer Academic Publishers)

Eichler, D., Livio, M., Piran, T. \& Schramm, D. N. 1989, Nature, 340, 126

Fenimore, E. E. et al. 1988, ApJ, 335, L71

Friedman, J. 1983, Phys. Rev. Lett., 51, 11

Goodman, J. 1986, ApJ, 308, L47

Goodman, J., Dar, A., \& Nussinov, S. 1987, Ap. J. Letters, 314, L7

Haensel, P., Paczyński, B., \& Amsterdamski, P. 1991, ApJ, 375, 209

Higdon, J. C. \& Lingenfelter, R. E. 1990, ARAA, 28, 401

Kochanek, C. S. 1992, ApJ, submitted

Lincoln, C. W. \& Will, C. M. 1990, Phys. Rev., D42, 1123

Mao, S. \& Paczynski, B. 1992, ApJ (Lett.), in press

Meegan, C. A. et al. 1992, Nature, 355, 143

Messina, D. C. \& Share, G. H. 1992, Proc. Huntsville GRO Meeting, eds. W. S. Paciesas \& G. J. Fishman, in press

Murakami, T., et al. 1988, Nature, 335, 234

Murakami, T., et al. 1991, Nature, 350, 592

Narayan, R. \& Goodman, J. 1989, Proc. NATO Advanced Research Workshop on "Theory of Accretion Disks", eds. F. Meyer, W. J. Duschl, J. Frank \& E. MeyerHofmeister, p. 231

Narayan, R., Piran, T. \& Shemi, A. 1991, ApJ, 379, L17 
Paczyński, B. 1986, ApJ, 308, L51

Paczyński, B. 1990, ApJ, 363, 218

Paczyński, B. 1991, Acta Astron., 41, 257

Parker, E. N. 1966, ApJ, 145, 811

Phinney, E. S. 1991, ApJ, 380, L17

Piran, T. 1990, in Supernovae, eds. J. C. Wheeler, T. Piran \& S. Weinberg, World Sci. Publ., Singapore

Piran, T. 1992, ApJ (Lett.), in press

Piran, T., Narayan, R. \& Shemi, A. 1992, Proc. Huntsville GRO Meeting, eds. W. S. Paciesas \& G. J. Fishman, in press

Ruderman, M. A. 1975, Ann. N Y Acad. Sci., 262, 164

Schaefer, B. E. 1990, Los Alamos Workshop on Gamma-Ray Bursts, July 29 - Aug. 3,1990

Schmidt, W. K. H. 1978, Nature, 271, 525

Shemi, A. \& Piran, T. 1990, ApJ, 365, L55

Taylor, J. H. \& Weisberg, R. M. 1989, ApJ, 345, 434

Trimble, V. 1991, Cont. Phys., 32, 103

van Kerkwijk, M. H. et al. 1992, Nature, 355, 703

Vogt, R. 1992, in Proc. MG6, eds. K. Sata \& T. Nakamura, World Sci. Publ., Singapore, in press 